\title{
Predictors and Mechanisms of Atrial Fibrillation in Patient with Hypertrophic Cardiomyopathy
}

Claire E Raphael* MD PhD, Alphonsus C Liew* MBBS MRCP, Frances Mitchell MBBS, Gajen Sunthar Kanaganayagam PhD MRCP, Elisa Di Pietro MD ${ }^{4}$, Simon Newsome MSc ${ }^{4}$, Ruth Owen $\mathrm{MSc}^{4}$, John Gregson $\mathrm{PhD}^{4}$ Robert Cooper MBBS PhD, Fouad R Amin MBBCh $\mathrm{MD}^{5}$, Peter Gatehouse PhD, Vassilis Vassiliou MA MRCP ${ }^{3}$, Sabine Ernst MD ${ }^{1}$, Rory O’Hanlon $\mathrm{MD}^{1}$, Michael Frenneaux $\mathrm{PhD}^{3}$, Dudley J Pennell MD, Sanjay K Prasad MD FRCP

*Joint first author

Running title: Predictors and mechanisms of AF in HCM

\author{
Affiliations \\ ${ }^{1}$ NIHR Cardiovascular Biomedical Research Unit, Royal Brompton Hospital, London, UK \\ ${ }^{2}$ Imperial College NHS Foundation Trust \\ ${ }^{3}$ University of East Anglia, UK \\ ${ }^{4}$ London School of Hygiene \& Tropical Medicine, UK \\ ${ }^{5}$ Frimley Park Hospital, UK
}

\section{Funding}

The work was supported by CORDA and the Rosetrees Foundation Trust, United Kingdom. Dr. Raphael supported by a British Heart Foundation Clinical Research Training Fellowship, United Kingdom.

\section{Disclosures}

Dr. Pennell has received a grant from Siemens; is the Director of and shareholder with CVIS; and has received personal fees from Bayer. Dr.Prasad has received personal fees from BayerSchering. All other authors have reported that they have no relationships relevant to the contents of this paper to disclose.

\section{Address for correspondence}

Dr Claire Raphael

Royal Brompton Hospital

Sydney Street

SW3 6NP

London

United Kingdom

Claire.raphael@gmail.com

tel: +442073528121

fax: +442073518816 


\begin{abstract}
Atrial fibrillation (AF) in hypertrophic cardiomyopathy (HC) is associated with significant symptomatic deterioration, heart failure and thromboembolic disease. There is a need for better mechanistic insight and improved identification of at risk patients.: We used CMR to assess predictors of $\mathrm{AF}$ in $\mathrm{HC}$, in particular the role of myocardial fibrosis. Consecutive patients with HC referred for CMR 2003-2013 were prospectively enrolled. CMR parameters including left ventricular volumes, presence and percentage of late gadolinium enhancement in the left ventricle (\%LGE) and left atrial volume index (LAVi) were measured. Overall, 377 patients were recruited (age $62 \pm 14$ years, $73 \%$ men). Sixty-two patients $(16 \%)$ developed new-onset AF during a median follow up of 4.5 (IQR 2.9 - 6.0) years. Multivariable analysis revealed \%LGE [hazard ratio (HR) 1.3 per 10\% (CI: 1.0-1.5; p=0.02), LAVi [HR 1.4 per $\left.10 \mathrm{~mL} / \mathrm{m}^{2}(1.2-1.5 ; \mathrm{p}<0.001)\right]$, age at $\mathrm{HC}$ diagnosis, non-sustained ventricular tachycardia and diabetes to be independent predictors of AF. We constructed a simple risk prediction score for future AF based on the multivariable model with a Harrell's C-statistic of 0.73. In conclusion, the extent of ventricular fibrosis and LA volume independently predicted AF in patients with $\mathrm{HC}$. This finding suggests a mechanistic relation between fibrosis and future AF in HC. CMR with quantification of fibrosis has incremental value over LV and LA measurements in risk stratification for AF. A risk prediction score may be used to identify patients at high risk of future AF who may benefit from more intensive rhythm monitoring and a lower threshold for oral anticoagulation.
\end{abstract}

\title{
Keywords
}

Hypertrophic cardiomyopathy, atrial fibrillation, prognosis, cardiovascular magnetic resonance, CMR, outcome 


\section{Introduction}

Patients with hypertrophic cardiomyopathy $(\mathrm{HC})$ have a 10-fold risk of developing atrial fibrillation (AF) compared to the general population ${ }^{1,2}$ and these patients have a high risk of stroke $^{3}$ and heart failure ${ }^{3-5}$. Our current practice is periodic ambulatory ECG monitoring and monitoring of symptoms, however stroke may be the first clinical presentation of AF. Identifying patients at risk of AF would allow more targeted, personalized treatment and more judicious use of continuous ambulatory monitoring. There are a number of potential contributory structural factors that may lead to development of AF, including left atrial dilatation $^{6}$, atrial myocardial fibrosis ${ }^{7,8}$ and myocardial ischaemia ${ }^{9}$. However, the value of these parameters in predicting development of $\mathrm{AF}$ in $\mathrm{HC}$ remains unclear. Cardiovascular magnetic resonance (CMR) allows quantification of fibrosis burden, accurate ventricular and atrial volume measurement and detection of myocardial ischemia ${ }^{10}$. Accordingly, we aimed to assess the prognostic value of CMR parameters to predict the development of $\mathrm{AF}$ in $\mathrm{HC}$.

\section{Methods}

From December 2003 to April 2013, consecutive patients referred with a diagnosis of known or suspected HC underwent a CMR scan including myocardial perfusion and late gadolinium enhancement at the Royal Brompton Hospital in London UK. All patients met the American College of Cardiology Foundation/American Heart Association (ACCF/AHA) diagnostic criteria for $\mathrm{HC}^{11}$. Exclusion criteria were previous or current $\mathrm{AF}$, metabolic diseases resulting in $\mathrm{HC}$ phenocopies such as Anderson-Fabry disease, significant primary valvular disease (excluding mitral regurgitation secondary to systolic anterior motion of the mitral valve), patients with contra-indication to CMR study including presence of an implantable cardioverter defibrillator (ICD) or pacemaker and patients with severe untreated hypertension. The study was approved by the Institutional Ethics Committee. Written informed consent was obtained from all study patients. 
CMR imaging was performed on a $1.5 \mathrm{~T}$ clinical scanner (Siemens Sonata/Avanto, Erlangen, Germany. Anatomic imaging was performed using Half-Fourier Acquisition Single-shot Turbo spin Echo (HASTE) sequences and steady-state free precession sequences were used to obtain cine images in standard two-, three- and four-chamber long axis views, with subsequent contiguous short-axis cines from the mitral annulus to the apex of the heart. The left atrium was assessed in two and four chamber long axis views.

Late gadolinium enhancement (LGE) images were acquired 10 minutes after the administration of intravenous gadolinium contrast agent (Gadovist or Magnevist, BayerSchering, Berlin, Germany, $0.1 \mathrm{mmol} / \mathrm{kg}$ ) in both long- and short- axes with a breath-hold inversion-recovery gradient echo sequence. The inversion time was adjusted to ensure adequately nulled myocardium and images were obtained in two orthogonal phase-encoding directions to allow exclusion of artefact.

Myocardial first-pass perfusion imaging was performed using a saturation-recovery prepared dual-sequence approach with center-out hybrid echoplanar imaging (EPI) using the following typical sequence parameters: fat saturation pulse, composite $90^{\circ}$ saturation preparation pulse for each slice, $28^{\circ}$ readout pulse, repetition time $5.1 \mathrm{~ms}$, echo time $1.1 \mathrm{~ms}$, echo train length 4 , field of view $360 \times 288 \mathrm{~mm}$, base resolution $160 \times 160$, slice thickness 8 $\mathrm{mm}$. Three short axis images were acquired every cardiac cycle for a total of 30 cycles at peak adenosine hyperemia. Adenosine was infused at $140 \mathrm{mcg} / \mathrm{kg} / \mathrm{min}$ for 4 minutes and symptoms, heart rate and blood pressure were monitored. At peak hyperemia, a bolus of gadolinium contrast (Magnovist or Gadovist, Bayer-Schering, Berlin, Germany, 0.1 $\mathrm{mmol} / \mathrm{kg}$ ) was rapidly injected, followed by a saline bolus.

A commercially available program (CMR Tools, Cardiovascular Imaging Solutions, London) was used to measure left ventricular volumes, stroke volume and ejection fraction. Left atrial (LA) area and length were carefully measured in two- and four-chamber views at 
end ventricular systole. The LA area was measured by tracing the atrial endocardial border, excluding the pulmonary veins, LA appendage and mitral recess and the LA volume was then calculated using the biplane area-length method ${ }^{12}$ and indexed to body surface area (LAVi).

Ventricular LGE was quantified using the "full width half maximum" method ${ }^{13}$ using dedicated imaging software (CMR42, Circle Cardiovascular Imaging, Calgary, Canada) and presented as a percentage of total LV mass (\%LGE). All analyses were performed by experienced operators blinded to patient outcome.

An inducible perfusion defect was reported to be present if hypointense signals were demonstrated in the subendocardial or transmural regions of the LV wall, persisting for three or more frames from when contrast is first visualized in the LV myocardium on stress images but not in concurrent rest images. Papillary muscles were not included in the perfusion assessment. A summed difference score (SDS) ${ }^{14,15}$ utilizing the ACC/AHA 17-segment mode ${ }^{16}$ but excluding the cardiac apex, was used. The following scores were used to rate each segment at rest and stress: 0 - no defect, 1 - inducible perfusion defect $<50 \%$ of wall thickness, 2 - inducible perfusion defect $>50 \%$ of wall thickness. The SDS was derived by subtracting the rest score from the stress score.

The endpoint was defined as a new diagnosis of AF, either paroxysmal or persistent. Paroxysmal AF was defined as AF that terminated within 7 days and persistent AF was defined as AF that lasted longer than 7 days. AF was considered to be present if a diagnosis of new AF was documented in the patient record or an ECG demonstrating AF was recorded. Where a cardiac implantable electronic device was used to diagnose $\mathrm{AF}$, a minimum duration of $>30$ s was used for the diagnosis. Where the presence of AF was in doubt due to unclear documentation (e.g. documentation of "possible AF on Holter monitor"), original ECG records were obtained and if necessary, an independent cardiologist was enlisted as a final adjudicator. Ambulatory ECG monitoring during follow-up was performed at the 
cardiologist's discretion. The majority of patients had a yearly Holter monitor.

Patients' vital statuses (alive/dead) were checked at six-monthly intervals via the UK National Strategic Tracing Service. Patients were followed up via a combination of telephone and postal questionnaires as well as review of medical records from primary and secondary care at 6-month intervals. The received data was assessed by a clinician for documentation of any $\mathrm{AF}$ events.

Baseline characteristics are presented as frequency $(\%)$ for categorical data and mean \pm standard deviation (SD) or median (IQR) for continuous data as appropriate. Baseline variables were compared between groups using Fisher's exact test for categorical variables and Mann-Whitney U test for continuous variables. Kaplan-Meier survival curves were generated and compared using the log-rank test. Univariable Cox proportional hazards models were used to test the association between baseline covariates and subsequent AF. Results were presented as hazard ratios and $95 \%$ confidence intervals. A multivariable risk prediction model was built using a forward stepwise selection procedure. A p-value $<0.05$ was considered significant. Harrell's C statistic was used to determine LAVi, \%LGE and age at diagnosis cut-off points that provided the best discrimination between the AF and no AF populations and Kaplan-Meier curves were generated using these cut-off points. A risk score was generated using the coefficients from the multivariable Cox model. Missing data was dealt with using multiple imputation by chained equations. The imputation models included the primary outcome, the Nelson-Aalen estimator of the cumulative hazard function and baseline variables with no missing data. Ten imputation sets were generated and mean values averaged over these sets were imputed for the model selection process. The coefficients of the risk prediction model were estimated by averaging over the 10 imputations using Rubin's rule $^{17}$. Statistical analysis was performed using Stata 15.

\section{Results}


A total of 377 patients (mean age $62 \pm 14,73 \%$ male) were recruited. Exclusions were prior or current $\operatorname{AF}(n=18,5 \%)$, inadequate baseline data $(n=5,1 \%)$ and patients lost to follow up (n=6, 2\%), giving a final population of 348 patients (Figure 1, Table 1).

The median follow-up period was 4.5 years $(2.9,6.0)$ with a total of 1532 patientyears of follow-up. During follow-up, sixty-two patients (16\%) developed new AF. The incidence of AF was 4.0 (CI: 3.2-5.2) per 100 person-years. There were 22 deaths during follow-up including 1 stroke death, 6 heart failure deaths and 2 sudden cardiac deaths. Six patients $(2 \%)$ had an ischemic stroke during follow-up, none of whom had AF at baseline. Two were subsequently diagnosed with AF during workup for stroke.

This was a real-world study design and no follow-up strategy for AF detection was prespecified across the cohort. The majority of patients underwent yearly annual ambulatory ECG monitoring. Two subsets had standardized follow-up: patients under follow-up at the Royal Brompton Hospital, who underwent regular annualized ambulatory ECG monitoring (53 patients, 15\%), and those with subsequently implanted devices (48 patients with ICD or pacemaker (14\%)). New AF was diagnosed in 14 (26\%) Brompton patients and $14(29 \%)$ patients with ICD/pacemaker. In the rest of the cohort, $36(15 \%)$ had new AF. Neither subset were found to be predictive of AF on univariable analysis $(p=0.35$ and $p=0.90$, respectively), however the study was not powered to assess this.

Univariable and multivariable analyses for predictors of AF are shown in Table 2. .LAVi and \%LV LGE were independently predictive of AF. The range of LAVi and \%LV LGE and their corresponding hazard ratios and estimated 5-year event rates are described in Table 3 and Figure 2 respectively. Example CMR images are shown in Figure 4. The cut-off points for \%LGE and LAVi which maximized discrimination of the multivariable risk prediction model were found to be $16 \%$ and $52 \mathrm{~mL} / \mathrm{m}^{2}$ respectively. These were used to generate Kaplan-Meier curves (Figure 3). Example CMR images are shown in Figure 4. At 5 
years, patients with LGE $>16 \%$ were more than twice as likely to develop AF compared to LGE $\leq 16 \%$ (23\% vs. $12 \% ; \mathrm{p}=0.005$, Figure $3 \mathrm{a})$ and patients with $\mathrm{LAVi}>52 \mathrm{~mL} / \mathrm{m}^{2}$ were more than three times as likely to develop AF compared to LAVi $\leq 52 \mathrm{~mL} / \mathrm{m}^{2}(25 \%$ vs. $8 \%$; $\mathrm{p}<0.0001$, Figure $3 \mathrm{~b}$ ). Based on the multivariable model the five-year risk of development of AF can be predicted as follows:

5 Year Risk $=1-0.997 \mathrm{e}(\mathrm{RS})$

Where $\mathrm{RS}=[0.030 \times \mathrm{LAVi}]+[0.023 \times \% \mathrm{LGE}]+[$ age at diagnosis of $\mathrm{HC} \times 0.031]+$ [0.679 (if NSVT)]+[0.918 (if Diabetic) $]$

$(\mathrm{RS}=$ risk score, $\mathrm{e}=$ exponential function $)$

The Harrell's C Statistic for this model was 0.73.

A simplified risk score model was constructed by dividing the population into lowrisk, medium-risk and high-risk groups based on the five factors predicting AF on multivariable analysis. Patients were considered low risk if they had 0 or 1 risk factor, medium risk if they had 2 factors and high risk if they had 3 or more risk factors. A score of 1 was given for each of the following: $\mathrm{LGE}>16 \%, \mathrm{LAVi}>52 \mathrm{~mL} / \mathrm{m} 2$, diabetes, presence of NSVT and age at diagnosis $>53$ years. Figure $3(\mathrm{c})$ shows the cumulative incidence of AF stratified by risk-group. The HR for developing AF for the medium- and high-risk groups compared to the low risk group were 4.9 (CI: 2.1-11.2) and 12.6 (5.5-29.0) respectively. The Harrell's C Statistic for the simplified model was 0.73 , demonstrating similar power of discrimination to the full risk model. 


\section{Discussion}

Patients with $\mathrm{HC}$ who develop AF are known to be at increased risk for heart failure and thromboembolic events, including stroke. We demonstrated that the extent of myocardial fibrosis and LA volume determined by CMR were strong independent predictors of AF. Our results suggest that myocardial replacement fibrosis may have a mechanistic role in development of AF. We formulated a risk prediction that may be used to identify high risk patients. Compared to those with minimal or no LGE and LAVi $40 \mathrm{~mL} / \mathrm{m}^{2}$, patients with moderate \%LGE (10\%) and LAVi $60 \mathrm{~mL} / \mathrm{m}^{2}$ were more than twice as likely to develop AF whereas patients with extensive \%LGE (40\%) and a LAVi of $\geq 80 \mathrm{ml} / \mathrm{m}^{2}$ had more than an 8 fold risk of developing AF during follow-up. CMR allows identification of high-risk patients who may benefit from more intensive follow up, perhaps with wearable digital ambulatory devices, and in whom there should be a low threshold for oral anticoagulation.

Our data suggest a potential causal relationship between \%LGE and AF in HC. Other variables closely linked to $\mathrm{HC}$ disease severity such as maximum wall thickness, LV volumes, resting LV outflow tract gradient and LV ejection fraction were not significant predictors of AF, suggesting that the relationship between AF and \%LGE is unlikely to simply reflect an increased prevalence of AF in more severe disease. Anti-fibrotic therapies may therefore reduce the incidence of new $\mathrm{AF}$ in $\mathrm{HC}$.

The initiation and maintenance of AF is complex (Figure 5). LV fibrosis may lead to increased LV filling pressures and a chronically increased LA pressure, leading to LA dilatation $^{18}$. Fibrosis within the left atrial wall may additionally interfere with local atrial conduction $^{19-21}$. We found that ventricular LGE remained a significant predictor of AF following adjustment for LA volume, suggesting that LA stretch alone does not fully explain the development of AF. Measurement of LA fibrosis is challenging due to the thin-walled left atrium, partial volume effects and $\operatorname{artefact}^{22}$, although recent efforts to produce accurate and 
reproducible LA fibrosis measurements have been promising ${ }^{23,24}$. While it was not possible to assess the amount of atrial fibrosis directly in this study, we would suggest that patients with extensive ventricular fibrosis are likely to also have a greater degree of atrial fibrosis ${ }^{21}$. However, ventricular fibrosis may act as a marker of more severe or progressive HC leading to increased risk of $\mathrm{AF}$ rather than a causal relationship ${ }^{25}$.

In contrast to previous work ${ }^{6,8}$, we found LA volume and LGE to be independently predictive of AF. This is likely due to the greater number of events in our cohort, however we also employed different methods for quantification of LGE (full width half maximum rather than $6 \mathrm{SD})^{13}$. To our knowledge, this is the largest study to date demonstrating the extent of LV LGE to be an independent predictor of $\mathrm{AF}$ in $\mathrm{HC}^{8,26}$. In keeping with previous work, we demonstrated $\mathrm{LAVi}^{1}$ and diabetes ${ }^{27,28}$ to be independent predictors of $\mathrm{AF}$. We extended this work to formulate a risk prediction model that could easily be employed in day-to-day clinical practice to identify high-risk patients.

We would suggest that frequent use of ambulatory ECG monitoring or patient activated event monitoring may be of benefit in patients with a severely dilated LA and extensive LGE who are currently in sinus rhythm. Wearable electronic devices capable of ECG provide an opportunity for continuous monitoring without implantation of loop recorders and may be the modality of choice going forward, although the sensitivity of these devices in capturing AF is outside the scope of our study. Patients at high risk may even benefit from prophylactic anticoagulation, although this requires formal evaluation on a risk/benefit basis. Interestingly, patients who went on to develop AF had a higher prevalence of beta blocker use at baseline than those who did not develop AF (68\% vs 52\%, p=0.02), suggesting that beta blockers may not be effective at preventing future $\mathrm{AF}$ in $\mathrm{HC}$, although this cannot be concluded from observational data.

Study Limitations: This was a single-center study in a tertiary referral center for CMR 
and may therefore be subject to referral bias. This was partly mitigated since referrals were from a broad source including our cardiomyopathy clinical service and a network of referring hospitals. As CMR was used in this study, patients with more advanced disease may have been excluded due to presence of an ICD or pacemaker. The mean age of our cohort was $62 \pm 14$ years and our cohort may therefore reflect more established disease. Our findings and risk score will need to be validated in other cohorts.

Patients did not have continuous ECG monitoring, nor was frequency of monitoring standardized. Episodes of asymptomatic paroxysmal AF would not have been captured and the true prevalence of AF in our cohort is therefore likely underestimated. Similarly, the 5year risk of $\mathrm{AF}$ as predicted by our model may be an underestimate. However, this is representative of real-world practice and is in keeping with the methodology of previous studies. Importantly, any decisions on management are likely to be based on a similar level of monitoring. We did not find a difference in rates of AF in patients followed up at our institution (where yearly $24 \mathrm{~h}$ ECG monitors were routinely performed) or those with devices capable of continuous rhythm recording compared to the remainder of the population. T1 mapping was not available at the outset of the study and therefore not performed. Our sequences were not optimized for pulmonary vein assessment so pulmonary vein morphology was not assessed.

In conclusion, both LA volume and \%LGE had independent prognostic value in prediction of AF in $\mathrm{HC}$. This suggests a potential mechanistic role for myocardial fibrosis in the development of AF in HC. In addition to risk stratification for sudden cardiac death, CMR allows identification of patients at high risk of future AF, allowing closer clinical follow-up and monitoring with the potential to introduce prophylactic anticoagulation therapy in those at highest risk. 


\section{References}

1. Guttmann OP, Rahman MS, O’Mahony C, Anastasakis A, Elliott PM. Atrial fibrillation and thromboembolism in patients with hypertrophic cardiomyopathy: systematic review. Heart 2014;100:465-472.

2. Davis RC, Hobbs FDR, Kenkre JE, Roalfe AK, Iles R, Lip GYH, Davies MK. Prevalence of atrial fibrillation in the general population and in high-risk groups: the ECHOES study. Europace 2012;14:1553-1559.

3. Olivotto I, Cecchi F, Casey SA, Dolara A, Traverse JH, Maron BJ. Impact of atrial fibrillation on the clinical course of hypertrophic cardiomyopathy. Circulation $2001 ; 104: 2517-2524$.

4. Tian T, Wang Y, Sun K, Wang J, Zou Y, Zhang W, Bao J, Zhu L, Shen H, Hui R, Zhou X, Song L. Clinical profile and prognostic significance of atrial fibrillation in hypertrophic cardiomyopathy. Cardiology 2013;126:258-264.

5. Robinson K, Frenneaux MP, Stockins B, Karatasakis G, Poloniecki JD, McKenna WJ. Atrial fibrillation in hypertrophic cardiomyopathy: a longitudinal study. J Am Coll Cardiol 1990;15:1279-1285.

6. Maron BJ, Haas TS, Maron MS, Lesser JR, Browning JA, Chan RH, Olivotto I, Garberich RF, Schwartz RS. Left atrial remodeling in hypertrophic cardiomyopathy and susceptibility markers for atrial fibrillation identified by cardiovascular magnetic resonance. Am J Cardiol 2016;113:1394-1400.

7. Papavassiliu T, Germans T, Flüchter S, Doesch C, Suriyakamar A, Haghi D, Süselbeck T, Wolpert C, Dinter D, Schoenberg SO, Van AC, Borggrefe M. Journal of Cardiovascular Magnetic CMR findings in patients with hypertrophic cardiomyopathy and atrial fibrillation. J Cardiovasc Magn Reson 2009;9:1-9.

8. Cochet H, Morlon L, Verge M-P, Salel M, Camaioni C, Reynaud A, Peyrou J, Ritter P, 
Jais P, Laurent F, Lafitte S, Montaudon M, Reant P. Predictors of future onset of atrial fibrillation in hypertrophic cardiomyopathy. Arch Cardiovasc Dis 2018;111:591-600. 9. Nishida K, Qi XY, Wakili R, Comtois P, Chartier D, Harada M, Iwasaki Y, Romeo P, Maguy A, Dobrev D, Michael G, Talajic M, Nattel S. Mechanisms of atrial tachyarrhythmias associated with coronary artery occlusion in a chronic canine model. Circulation 2011;123:137-146.

10. To ACY, Dhillon A, Desai MY. Cardiac magnetic resonance in hypertrophic cardiomyopathy. JACC Cardiovasc Imaging 2011;4:1123-1137.

11. Gersh BJ, Maron BJ, Bonow RO, Dearani JA, Fifer MA, Link MS, Naidu SS, Nishimura RA, Ommen SR, Rakowski H, Seidman CE, Towbin JA, Udelson JE, Yancy CW. 2011 ACCF/AHA guideline for the diagnosis and treatment of hypertrophic cardiomyopathy: executive summary: a report of the American College of Cardiology Foundation/American Heart Association Task Force on Practice Guidelines. Circulation 2011;124:2761-2796. 12. Lester SJ, Ryan EW, Schiller NB, Foster E. Best method in clinical practice and in research studies to determine left atrial size. Am J Cardiol 1999;84:829-832.

13. Flett AS, Hasleton J, Cook C, Hausenloy D, Quarta G, Ariti C, Muthurangu V, Moon JC. Evaluation of techniques for the quantification of myocardial scar of differing etiology using cardiac magnetic resonance. JACC Cardiovasc Imaging 2011;4:150-156.

14. Shah R, Heydari B, Coelho-Filho O, Murthy VL, Abbasi S, Feng JH, Pencina M, Neilan TG, Meadows JL, Francis S, Blankstein R, Steigner M, Carli M di, Jerosch-Herold M, Kwong RY. Stress cardiac magnetic resona62nce imaging provides effective cardiac risk reclassification in patients with known or suspected stable coronary artery disease. Circulation 2013;128:605-614.

15. Steel K, Broderick R, Gandla V, Larose E, Resnic F, Jerosch-Herold M, Brown K a, Kwong RY. Complementary prognostic values of stress myocardial perfusion and late 
gadolinium enhancement imaging by cardiac magnetic resonance in patients with known or suspected coronary artery disease. Circulation 2009;120:1390-1400.

16. Cerqueira MD, Weissman NJ, Dilsizian V, Jacobs AK, Kaul S, Laskey WK, Pennell DJ, Rumberger JA, Ryan T, Verani MS. Standardized myocardial segmentation and nomenclature for tomographic imaging of the heart. A statement for healthcare professionals from the Cardiac Imaging Committee of the Council on Clinical Cardiology of the American Heart Association. Circulation 2002;105:539-542.

17. Rubin DB. Multiple imputation for nonresponse in surveys. New York: John Wiley \& Sons; 1987.

18. Greenberg B, Chatterjee K, Parmley WW, Werner JA, Holly AN. The influence of left ventricular filling pressure on atrial contribution to cardiac output. Am Heart J 1979;98:742751.

19. Nattel S, Burstein B, Dobrev D. Atrial remodeling and atrial fibrillation: mechanisms and implications. Circ Arrhythm Electrophysiol 2008;1:62-73.

20. Burstein B, Nattel S. Atrial fibrosis: mechanisms and clinical relevance in atrial fibrillation. J Am Coll Cardiol 2008;51:802-809.

21. Sivalokanathan S, Zghaib T, Greenland G V, Vasquez N, Kudchadkar SM, Kontari E, Lu D-Y, Dolores-Cerna K, Geest RJ van der, Kamel IR, Olgin JE, Abraham TP, Nazarian S, Zimmerman SL, Abraham MR. Hypertrophic cardiomyopathy patients with paroxysmal atrial fibrillation have a high burden of left atrial fibrosis by cardiac magnetic resonance imaging. JACC Clin Electrophysiol 2019;5:364-375.

22. Appelbaum E, Manning WJ. Left atrial fibrosis by late gadolinium enhancement cardiovascular magnetic resonance predicts recurrence of atrial fibrillation after pulmonary vein isolation: do you see what I see? Circ Arrhythm Electrophysiol 2014;7:2-4. 23. Margulescu AD, Nuñez-Garcia M, Alarcón F, Benito EM, Enomoto N, Cozzari J, Chipa 
F, Fernandez H, Borras R, Guasch E, Butakoff C, Tolosana JM, Arbelo E, Camara O, Mont L. Reproducibility and accuracy of late gadolinium enhancement cardiac magnetic resonance measurements for the detection of left atrial fibrosis in patients undergoing atrial fibrillation ablation procedures. Europace 2019;21:724-731.

24. Benito EM, Carlosena-Remirez A, Guasch E, Prat-Gonzalez S, Perea RJ, Figueras R, Borras R, Andreu D, Arbelo E, Tolosana JM, Bisbal F, Brugada J, Berruezo A, Mont L. Left atrial fibrosis quantification by late gadolinium-enhanced magnetic resonance: a new method to standardize the thresholds for reproducibility. Europace 2017;19:1272-1279.

25. Nattel S. Molecular and cellular mechanisms of atrial fibrosis in atrial fibrillation. JACC Clin Electrophysiol 2017;3:425-435.

26. Pujadas S, Vidal-Perez R, Hidalgo A, Leta R, Carreras F, Barros A, Bayes-Genis A, Subirana MT, Pons-Llado G. Correlation between myocardial fibrosis and the occurrence of atrial fibrillation in hypertrophic cardiomyopathy: a cardiac magnetic resonance imaging study. Eur J Radiol 2010;75:e88-91.

27. Movahed M-R, Hashemzadeh M, Jamal MM. Diabetes mellitus is a strong, independent risk for atrial fibrillation and flutter in addition to other cardiovascular disease. Int J Cardiol 2005; 105:315-318.

28. Benjamin EJ, Levy D, Vaziri SM, D’Agostino RB, Belanger AJ, Wolf PA. Independent risk factors for atrial fibrillation in a population-based cohort. The Framingham Heart Study. JAMA 1994;271:840-844. 


\section{Figure and Table Legends}

Figure 1: Consort chart describing patient recruitment

Figure 2: Left atrial volume and LGE had independent prognostic values. 5-year KaplanMeier event rates for varying combinations of \%LGE and LAVi are displayed graphically.

Figure 3: Kaplan-Meier graphs comparing AF cumulative incidence between (a) $\% \mathrm{LGE} \leq 16 \%$ and $\% \mathrm{LGE}>16 \%$, (b) $\mathrm{LAVi} \leq 52 \mathrm{~mL} / \mathrm{m} 2$ and $\mathrm{LAVi}>52 \mathrm{~mL} / \mathrm{m} 2$ groups and (c) low-risk ( $\leq 1 \mathrm{RF})$, medium-risk (2 RF) and high-risk ( $\geq 3 \mathrm{RF}$ )groups based on the number risk factors (RF) with a score of 1 given for each of the following: $\mathrm{LGE}>16 \%, \mathrm{LAVi}>52 \mathrm{~mL} / \mathrm{m}^{2}$, diabetes, presence of NSVT and age at diagnosis $>53$ years, (d) $L G E \leq 16 \%$ and $\mathrm{LAVi} \leq 52 \mathrm{ml} / \mathrm{m}^{2}$ vs LGE $>16 \%$ and $\mathrm{LAVi}>52 \mathrm{ml} / \mathrm{m}^{2}$. Four patients did not have LGE quantification due to poor image quality.

Figure 4: Example CMR images. Measurement of the left atrium was performed in 2- and 4chamber views at end systole as described in panels A and B. Panels $\mathrm{C}$ and $\mathrm{E}$ show short axis LGE images of a low risk patient with no LGE enhancement. Panels D and F show a high risk patient with extensive LGE (arrowed) who went on to develop AF.

Figure 5 - Central illustration: Coumel's triangle adapted for HCM. The triangle describes the triggers, substrate and modulating factors that lead to atrial fibrillation. Risk factors found to be predictive of AF in our study are marked in bold and with an asterisk. Risk factors that have been previously described in AF in the general cardiology population but were not predictive in this study are in italics and marked with an obelisk $(\dagger)$.

Table 1: Baseline characteristics

Table 2: Univariable and multivariable analyses of AF predictors. *NSVT was not significant on univariable analysis as its presence was associated with younger age and less 
diabetes (which are protective factors for future AF). When entered in a multivariable model with these factors, it is unmasked as a significant predictor of future AF.

$\mathrm{HR}=$ Hazard ratio $; \mathrm{CI}=$ Confidence interval

Table 3: Hazard ratios (HR) and 95\% confidence intervals for varying combinations of \%LGE and LAVi compared to absent LGE and LAVi $40 \mathrm{~mL} / \mathrm{m}^{2}$.

Numbers are presented as HR (95\% confidence interval) 